FY05 LDRD Final Report Molecular

Engineering of Electrodialysis

Membranes 03-ERD-060

W. Bourcier, K. O'Brien, A. Sawvel, M. Johnson, K. Bettencourt, S. Letant, T. Felter, K. Langry, B. Wilson, J. Haslam, C. Schaldach, D. Sopchak

March 1, 2006 
This document was prepared as an account of work sponsored by an agency of the United States Government. Neither the United States Government nor the University of California nor any of their employees, makes any warranty, express or implied, or assumes any legal liability or responsibility for the accuracy, completeness, or usefulness of any information, apparatus, product, or process disclosed, or represents that its use would not infringe privately owned rights. Reference herein to any specific commercial product, process, or service by trade name, trademark, manufacturer, or otherwise, does not necessarily constitute or imply its endorsement, recommendation, or favoring by the United States Government or the University of California. The views and opinions of authors expressed herein do not necessarily state or reflect those of the United States Government or the University of California, and shall not be used for advertising or product endorsement purposes.

This work was performed under the auspices of the U.S. Department of Energy by University of California, Lawrence Livermore National Laboratory under Contract W-7405-Eng-48. 


\title{
FY05 LDRD Final Report Molecular Engineering of Electrodialysis Membranes 03-ERD-060 1
}

\begin{tabular}{lll}
\multicolumn{3}{c}{ William Bourcier, Principal Investigator } \\
& \multicolumn{1}{c}{ Co-Investigators: } & \\
Kevin O'Brien & Sonia Letant & Jeff Haslam \\
April Sawvel & Tom Felter & Charlene Schaldach \\
Mackenzie Johnson & Kevin Langry & David Sopchak \\
Kerry Bettencourt & Bill Wilson &
\end{tabular}

\begin{abstract}
Using a combination of modeling and experimental work we have developed a new method for purifying water that uses less energy than conventional methods and that can be made selective for removing targeted contaminants. The method uses nanoporous membranes that are permselective for anion or cation transfer. Ion selectivity results from double layer overlap inside the pores such that they dominantly contain ions opposite in charge to the surface charge of the membrane. Membrane charge can be adjusted through functionalization. Experiments confirm membrane permselectivity and overall energy use less than that for conventional electrodialysis. The nanoporous membranes are used in a conventional electrodialysis configuration and can be incorporated in existing electrodialysis systems without modification. The technology merits further development and testing in real systems, and could result in a significant reduction in water treatment costs.
\end{abstract}

\section{Introduction/Background}

Our project goal was to develop a more detailed understanding of the physics of aqueous ion transport through nanoporous membranes and use this understanding to develop new energy efficient and element selective membranes for use in water treatment. The project included four components: (1) modeling work aimed at predicting the electrostatic potentials in the vicinity of charged nanoengineered materials such as nanoporous membranes, (2) synthesis and functionalization of nanoporous membranes, (3) experimental measurements of membrane transport properties, and (4) development of plans and cost estimates for pilot field demonstrations of a new technology for water treatment. Our plan was that at the conclusion of this project, we would have a new energy efficient and selective technology for water treatment available for field-testing.

We chose nitrate contamination as our first target for this technology because of the importance of this problem for California's water supply. However, our broader goal was a technology

\footnotetext{
${ }^{1}$ This work was performed under the auspices of the U. S. Department of Energy (DOE) by the University of California, Lawrence Livermore National Laboratory (LLNL) under Contract No. W-7405-Eng-48. The project (03-ERD-060) was funded by the Laboratory Directed Research and Development Program at LLNL.
} 
platform that could be used for removal of any targeted species e.g. arsenic, selenate, borate, lithium, mercury, chromate, etc., or applied to sea water desalination. Understanding the basic physics behind ion transport through nanopores as well as selective transport (e.g. pore gating) was a key part of the project that helped to insure that a wide range of applications would be possible.

We chose to develop a new type of electrodialysis technology versus other or new water treatment technologies in this project for several reasons. The most important is that electrodialysis lends itself to selective extraction, because the technique removes ions from water by pulling away the ions and leaving the water behind. We believed that in a two-year project, we could conceivably develop and synthesize a new type of membrane and place it in existing electrodialysis units with minimal re-engineering, thus increasing the likelihood of success. We also observed that existing electrodialysis is very energy inefficient, and that our nanoporous membranes were likely to improve upon energy efficiency, as well as selectivity. In addition, we predicted that an optimized electrodialysis technology could be made much more energy efficient than reverse osmosis, where water is removed and salt is left behind.

A key aspect of our approach is that we replaced the currently used solid copolymer membranes used in electrodialysis with porous materials that have pore sizes tuned to provide ion permselectivity. This was accomplished by taking advantage of "double layer overlap" that occurs in pores that have radii less than the electrical double layer thickness. A double layer forms when a charged surface is brought into contact with an aqueous electrolyte solution. The double layer is the zone adjacent to the surface that contains counter ions that are opposite in sign and balance the surface charge. The double layer thickness is the thickness of the zone containing the counter ions. The structure of the double layer is different than the structure of

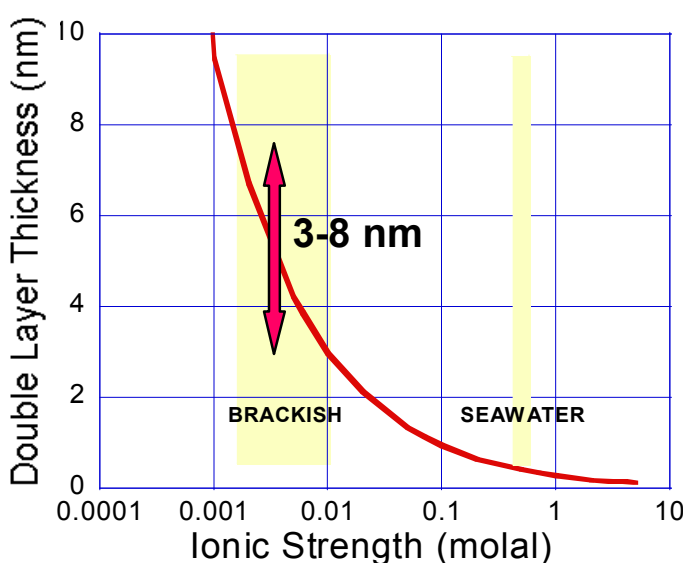

Figure 1. Double layer thickness as a function of ionic strength (for 1:1 electrolyte). bulk electrolyte solution, and generally has a lower dielectric constant. The double layer gets thinner as the ionic strength of the solution is increased, as shown in Figure 1.

When double layer overlap is present, it is difficult to move water through the pore without very high driving pressures. Therefore a membrane that has nanopores small enough to have double layer overlap will allow very little water transport. However, ion transport is allowed and can be driven with an electric field.

The pores in materials that have a negative surface charge will be filled with positive counter ions that balance the negative surface charge. Negative ions will be repelled from the pore volume. Ion transport through a pore in this condition driven by an electric field will be dominated by positive ions. The opposite situation exists for a positively charged pore. Thus the pores are "permselective" for ion 
transport. A porous material having a surface charge and pores small enough to develop double layer overlap will have favorable properties for use in electrodialysis separation. The material is permeable to ions of either positive or negative charge (depending on the material's surface charge), and will not allow significant water transport. The membrane can be used in a conventional electrodialysis configuration as shown in Figure 2.

We believed that it would take less energy to push ions through nanoporous membranes than through a solid functionalized polymer, the standard membrane material used for conventional electrodialysis. Moreover, by functionalizing the membranes with chemical groups with high affinities for targeted species (e.g. a nitrate binding group for nitrate) we believed it would be possible to generate an element-selective membrane. A technology with lower overall energy use coupled with ion selectivity would greatly lower the costs of treating contaminated water supplies. Our project goal was the development of this new electrodialysis technology.

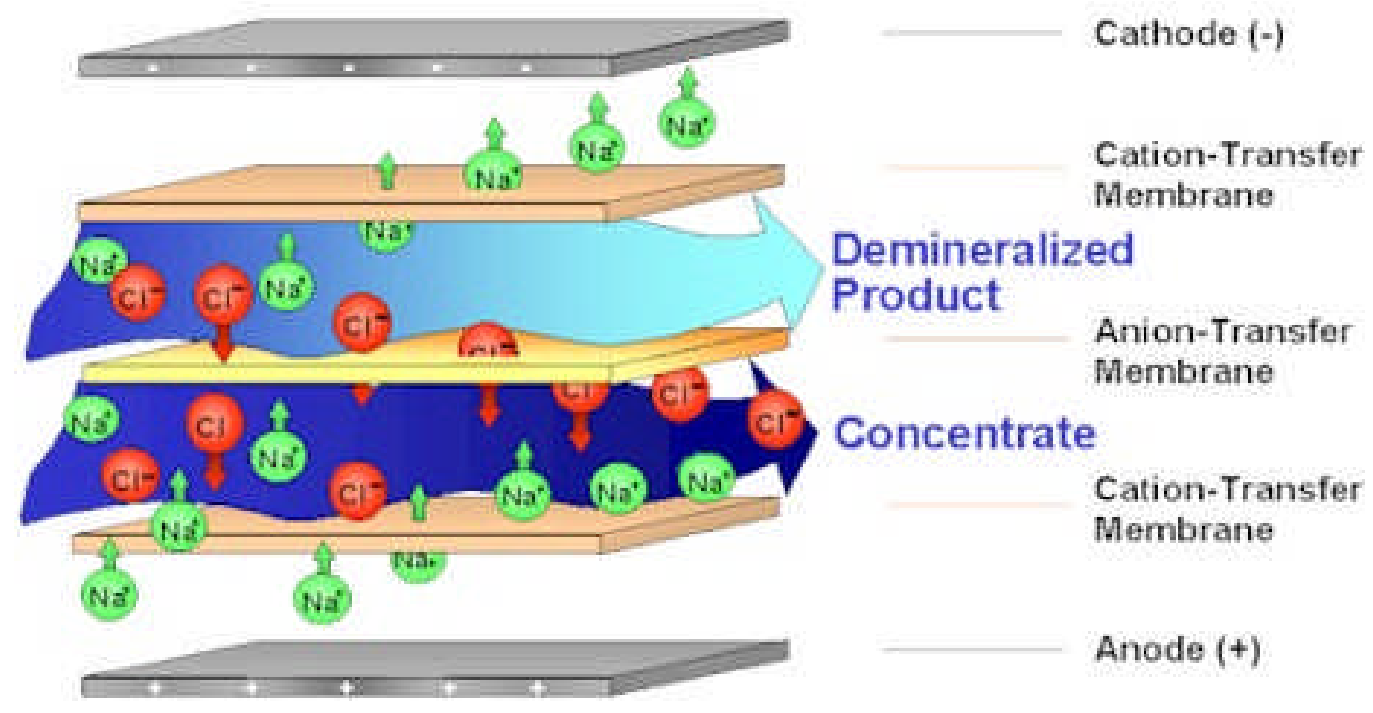

Figure 2. Typical electrodialysis configuration where a static electric field is applied across a stack of alternating cation and anion permeable membranes. As fluid is pumped through, alternate compartments become more or less saline due to ion migration through the membranes.

\section{Research Activities}

Our research activities included fabrication, functionalization, and characterization of membrane materials, testing of membranes in aqueous solutions, modeling of membrane processes, purchase and operation of a commercial electrodialysis unit, and planning for scale up and field operation of our new water cleanup technology. Many of the technical results and implications of this work are described in the attached publications, research proposals, and records of invention. The overview of the project motivations and accomplishments are summarized here. 


\section{$\underline{\text { Membrane fabrication }}$}

We originally selected two materials for use as membranes in this project: nanoporous polycarbonate and nanoporous alumina. Both materials are very durable given likely service conditions (low salinity near-neutral $\mathrm{pH}$ waters) and can be fabricated with controlled pore sizes down to the size range needed for our electrodialysis application $(5-16 \mathrm{~nm}$ or twice the double layer thickness; see Fig. 1). Polycarbonate has a negative surface charge at near-neutral pHs, and alumina has a positive charge. As a result, the two materials would provide a useful membrane pair; polycarbonate should allow cation transport and alumina should allow anion transport. In this project, we focused mainly on polycarbonate, based on our belief that polycarbonate would be a more durable membrane based on its plasticity, versus the more brittle alumina. Therefore polycarbonate would be the better choice for demonstration of proof of concept. Future work on alumina membranes may be warranted depending on whether polycarbonate can readily be functionalized to produce a positive charge.

Polycarbonate is made porous through ion track etching (Fig. 3). In this process, a high energy ion collides and passes through a polycarbonate film generating a trail of 'disturbed' material that is more soluble than bulk material. Etching with a basic solution such as sodium hydroxide dissolves away the disturbed material leaving a cylindrical pore. Commercial ion track etched polycarbonates (PCTE) are used in size exclusion filtration, and are a standard laboratory product (e.g Nucleopore filters). We carried out ion track etching here at LLNL in order to produce pore sizes smaller than the smallest commercially available (15-20 $\mathrm{nm}$ ), with an additional goal of making pores with more uniform diameters. SEM imaging of available commercial materials revealed significant nonuniformity of pores. Nominal $10 \mathrm{~nm}$ polycarbonates were seen to have pores up to $20 \mathrm{~nm}$ in diameter, with many pores greater than $30 \mathrm{~nm}$ in diameter. By fabricating our own materials, we had the option of generating pores of various geometries, other than simple cylindrical pores available in commercial materials, and generating very high pore densities in order to increase ion transport flux through the membrane in electrodialysis applications.

We used the accelerator in the LLNL ion beam laboratory (B-235) at 3.5 MV potential to accelerate $\mathrm{Xe}^{4+}$ and $\mathrm{Ar}^{3+}$ ions to kinetic energies of 14 and 10.5 $\mathrm{MeV}$. The ions were then allowed to collide with sheets of commercial polycarbonate of varying thicknesses (4-20 microns). The sheets were then etched at elevated temperatures in sodium hydroxide solutions, which preferentially etched

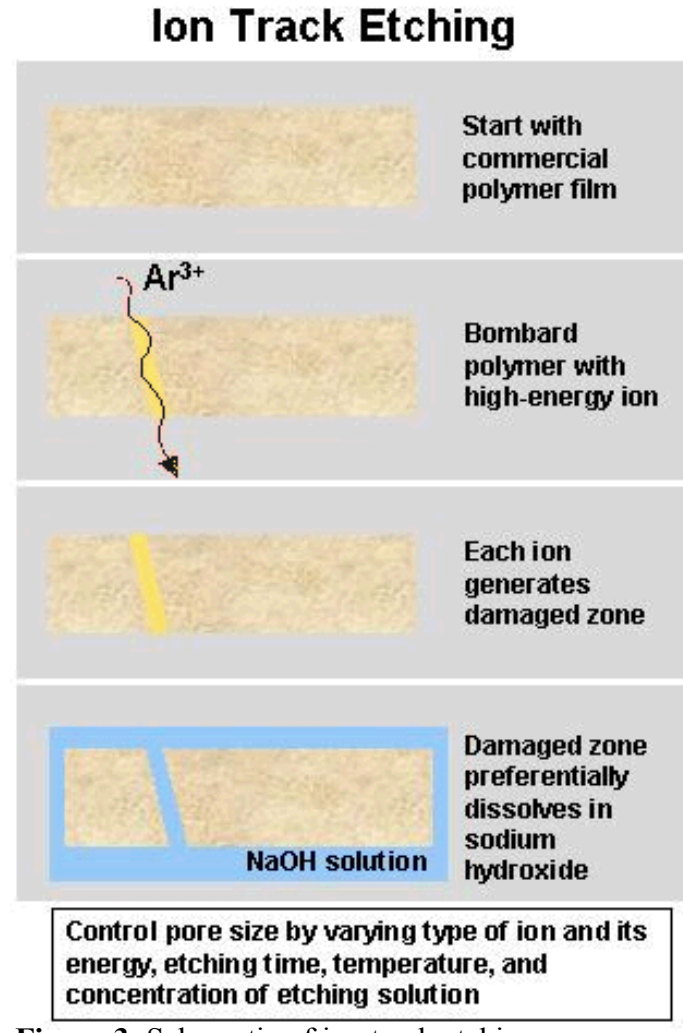

Figure 3. Schematic of ion track etching process. 
away the ion-damaged materials leaving a through-going pore. The pore diameter could be controlled by varying the etch time, temperature, and sodium hydroxide concentration. A matrix of tests was carried out in order to establish the optimum conditions for ion tracking in order to produce monodisperse, sub-10 nm, through-going pores. The pore sizes and size distribution was determined by imaging the polycarbonates using SEM. Pores smaller than $15 \mathrm{~nm}$ were imaged using field emission SEM in order to have the necessary resolution. The polycarbonate sheets were then used in membrane performance tests.

In carrying out this track etching work, we were in communication with two commercial suppliers. Osmonics (currently GE-Water Services) uses the nuclear reactor at Texas A\&M as an ion source for track etching and supplies most of the commercial PCTE materials available from scientific supply vendors in the U.S. The Osmonics materials were used in many of the membrane tests described below. In addition, we contacted and received materials from IT4IP, a small company associated with Université Catholique de Louvain in Belgium that fabricates PCTE using a cyclotron ion source. IT4IP provided us with polycarbonate membranes with very monodisperse and smaller pores $(<10 \mathrm{~nm})$ that were superior to any commercial materials available in the U.S. for our application. These membranes were also tested.

\section{Membrane functionalization}

Membrane functionalization refers to the process of attaching to the membrane surface and/or the inner pore surfaces chemical groups that either have a desired surface charge, or that interact with species in solution in a desirable way. For example, the surface could be functionalized with a group having a positive charge in order to make the membrane permselective for anion transport (pores would contain only negatively-charged species due to double layer overlap). Or the surface could be functionalized with groups that have high specificity for nitrate ions, such that the pore is dominantly filled with nitrate ions and would preferentially allow transport of nitrate through the pore. In this project,

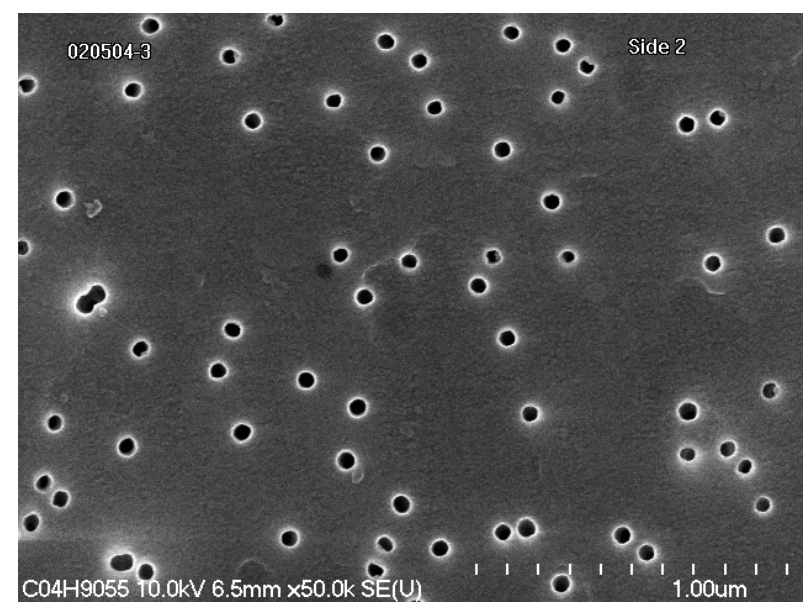

Figure 4. Ion track etched polycarbonate with pore diameters of about $50 \mathrm{~nm}$.

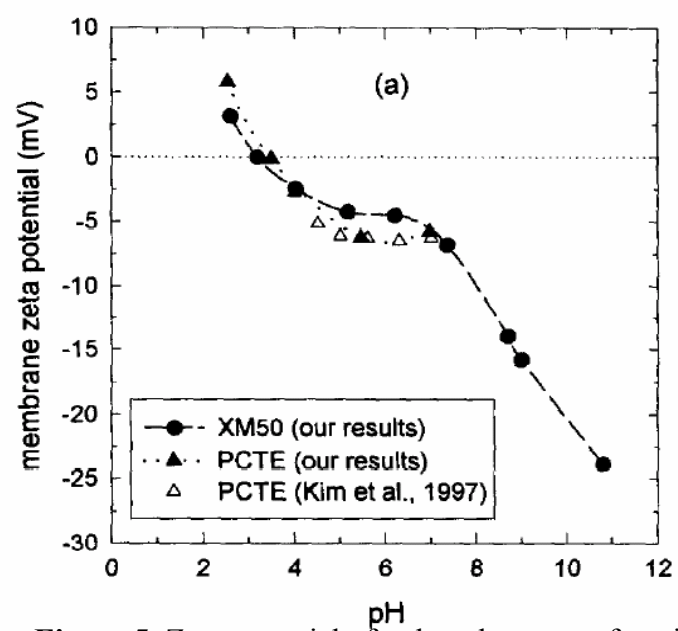

Figure 5. Zeta potential of polycarbonate as function of $\mathrm{pH}$. 
our efforts focused on functionalization to achieve a positive surface charge. Polycarbonate has an intrinsic negative surface charge in water above $\mathrm{pH} 3$ (Fig. 5) so that unfunctionalized polycarbonate works as a cation membrane without any further modification (however, adding a more strongly negative surface group made the membrane more efficient, see SLIP coating discussion below). Due to time and budget constraints, we did not carry out any functionalization work aimed at producing nitrate selectivity. This topic will be pursued through proposals to outside funding opportunities (see last section).

Generating a positive surface charge

Several attempts were made to modify the polycarbonate to produce a positive surface charge. The polycarbonate was treated with various silane-coupling agents with terminal quaternary amine groups, whose presence could be detected using infrared spectroscopy. Unfortunately, the attachments to polycarbonate were not stable and quickly desorbed when the membranes were placed in aqueous electrolyte solutions. This was verified with infrared spectroscopy. The surfaces were also examined with x-ray photoelectron spectroscopy (XPS) to look at relative amounts of nitrogen on the surface, with inconclusive results.

Membranes coated with self-assembled monolayers of organic polyelectrolytes were synthesized and applied by Luna Innovations using their patented ISAM process. Functionalized surfaces were confirmed using infrared spectroscopy. Unfortunately, these coatings also came off from the surface of the polycarbonate during testing, but only when a voltage was applied across the membrane.

In addition, several samples were functionalized using DICH/TEA/heptane, designed to put amines on the terminal phenol groups, and HxDA vapor or HxDA/heptane treatments to put an amine group on a cleaved carbonate group. These samples were synthesized late in the project and have not yet been tested.

A commercially available titanium-containing coupling agent, LICA 38J, (shown in Figure 6) did adhere well to polycarbonate even when voltages were applied to the functionalized membranes. The coating was applied from an aqueous solution and then crosslinked with UV radiation. The coating created a positively charged surface that performed well as an anion membrane in the bench-top testing.

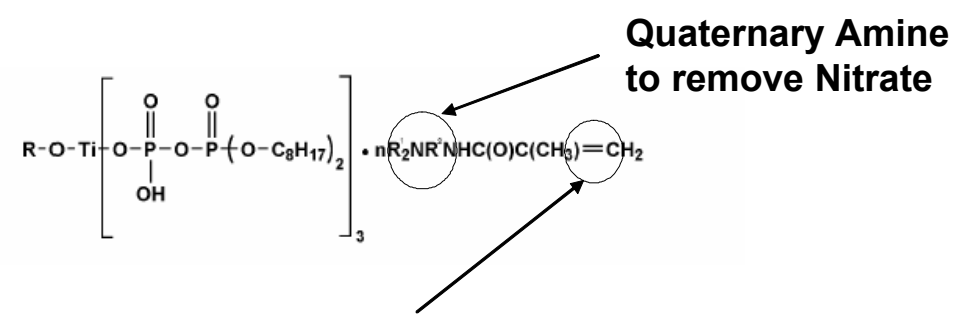

\section{Polymerize coating by reacting double bond}

Figure 6. LICA $38 \mathrm{~J}$ coupling agent. This titanate material had quaternary amines to provide a positive surface charge and produce anion permselectivity. 


\section{Enhancing the Negative Surface Charge}

The negative surface charge of polycarbonate was enhanced by applying LLNL's 'SLIP' process (solventless vapor deposition followed by In Situ polymerization). The SLIP process was originally developed for the fabrication of inertial confinement targets and is outlined in Figure 7. Polyimide layers $100 \mathrm{~nm}$ in thickness were deposited onto $10 \mathrm{~nm}$ porous polycarbonate membranes. Gas transport testing demonstrated that the pores were NOT blocked or bridged by the SLIP coating process. Zeta potential measurements illustrated that the SLIP coating increased the zeta potential of the surface from the as received sample value of $-25 \mathrm{mV}$ to values in excess of $-30 \mathrm{mV}$ (see Table 1 and discussion in next section). This enhanced zeta potential correlated with the improved performance of the SLIP coating polycarbonate, i.e. less energy needed to separate ions than 'as received' polycarbonate.
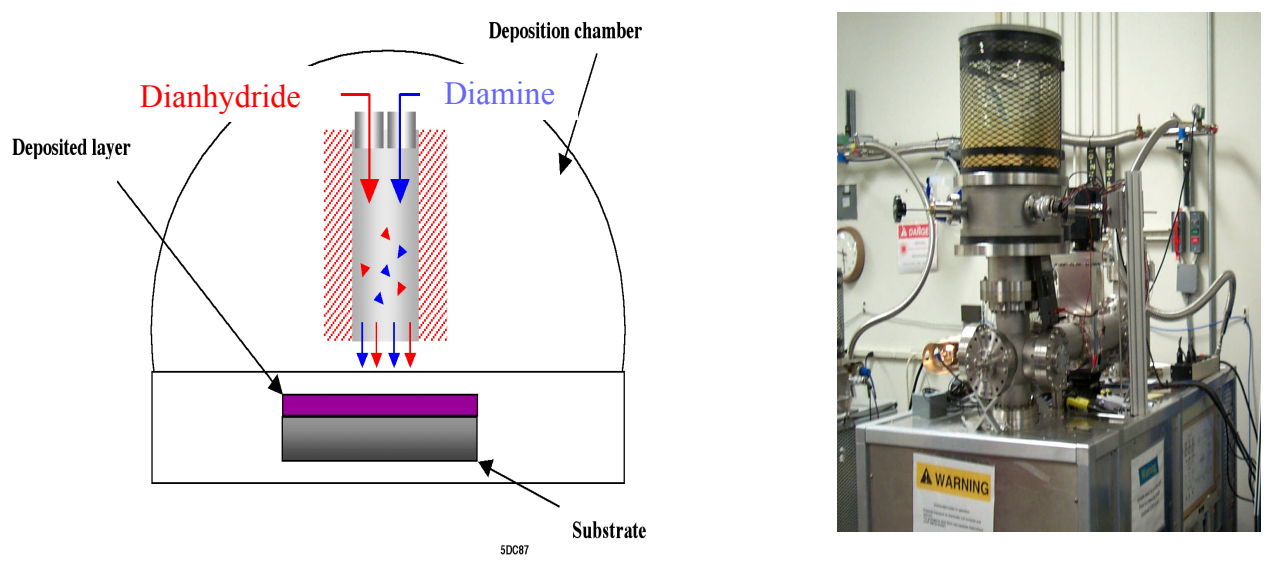

Figure 7. SLIP process for deposition of $100 \mathrm{~nm}$ polyimide film onto polycarbonate. Schematic on left, apparatus on right.

\section{Metalization}

We investigated the approach of coating the polycarbonate surface with a conductive metal layer as an alternate way to generate a surface charge. The idea is to externally apply a voltage using a wire connected to the metalized surface. In this way, the membrane can be charged as desired. An advantage is that if a membrane became fouled or coated with an unwanted material, the charge could be reduced or changed in polarity to expel the unwanted coating, potentially an effective cleaning method. The charge could also be used to oxidize or reduce materials, such as reducing nitrate to nitrogen gas, or perchlorate to chloride ions, both benign by-products. This would eliminate any waste stream associated with nitrate or perchlorate removal. Metalized membranes thus have key advantages over functionalized membranes for water treatment applications, and merit further work.

Both gold and platinum were vapor deposited on the polycarbonate after plasma etching to clean the surface. SEM imaging revealed that the metal layers did not cover the pore openings, but instead simply coated the membrane surface and partially penetrated the pore. This coating 
geometry is desirable because we believe that a through-going metalized pore would shield the electrostatic field inside the pore and reduce ion flux during electrodialysis.

Metalized polycarbonate membranes were tested repeatedly in the electrodialysis cell. In all cases, although the metal layers adhered to the surfaces when immersed in salt solution in the test cell, when a DC potential was applied, the layers consistently flaked off after only a few minutes in the DC field. This problem was not overcome with modifications to our metalization methods, such as varying plasma etching conditions to clean the polycarbonate prior to metal coating, using either gold and platinum coatings, varying the thickness of the metal layer, the use of a titanium stick layer, the use of both PVP-coated (poly-vinylpyrrolidone) and uncoated polycarbonates, and the use of polycarbonates from several different sources. Because of these results, the metalization task was put on hold and effort switched to chemical functionalization. In the long term, metalization would probably be preferable over functionalization for water treatment applications because of the good corrosion resistance of the metal layers, the ability to clean the membranes using an applied charge, the ease with which the membranes could be remetalized versus re-functionalized, and the fact that with metalization, no potentially harmful organic compounds could be leached from the membrane into the water supply, an important consideration for functionalized membranes used in water treatment plants.

\section{Measurements of surface charge}

As discussed above, the surface charge of the membranes is important in generating ion permselectivity in nanopores. The surface charge gives rise to a measurable electrokinetic property called 'zeta potential', which is the charge at the slip plane that separates the charged surface and associated counterions (double layer) from bulk electrolyte solution. The zeta potential should correlate with membrane permselectivity. For this reason, we carried out measurements of the streaming potential of the polycarbonate and alumina membranes using a Brookhaven Instruments EKA Electrokinetic Analyzer (BI-EKA) and used the results to calculate the zeta potentials of various membrane materials in several fluid compositions. For these measurements, samples of the membranes were placed in a cell in which an electrolyte solution is streamed over the surface of the membrane under investigation. As the electrolyte solution passes over the sample, a displacement of charge occurs as ions in the double layer are carried along. Because the double layer contains predominantly one type of charge (plus or minus), a small voltage develops along the surface parallel to the direction of fluid flow. The resulting "Streaming Potential / Streaming Current" is detected by means of electrodes placed at each end of the sample. The measured streaming potential can be used to compute the zeta potential of the material from a derivation that includes Poiseuille's formula, Poisson's equation, and Ohm's law. The relationship is given as:

$$
\Delta E=\frac{\varepsilon \zeta}{\eta \Omega} \Delta P
$$

where $\Delta E$ is the measured potential in volts, $\Delta P$ is the pressure gradient along the membrane surface, $\varepsilon$ is the dielectric constant of the solution, $\zeta$ is the zeta potential, $\eta$ is the viscosity of the solution, and $\Omega$ is the conductivity of the solution. 
The zeta potentials showed that polycarbonate membranes have a negative surface charge at near neutral $\mathrm{pH}$. Alumina has a positive surface charge (see Table 1). The negative surface charge of polycarbonate becomes less negative as the $\mathrm{pH}$ decreases, as expected. Our data differs from previous measurements of polycarbonate that showed positive zeta potential values at $\mathrm{pH}$ values

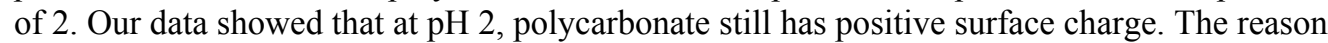
for this difference at present is unknown. We also found that the poly-vinylpyrrolidone (PVP) coating used to make hydrophilic polycarbonate membranes tends to make the surface charge more negative. We also found that the SLIP process produces a more negatively charged membrane, and is probably responsible for the increase in membrane performance in electrodialysis tests. The fact that the polycarbonate membranes act as cation-selective membranes is consistent with these measured negative surface charges.

Table 1. Zeta potentials of membranes determined from streaming potential measurements. Sample $\mathrm{pH}$ nominal $\quad \mathrm{pH}$ meas Zeta Pot. Std Dev

$\begin{array}{lllll}\text { 100nm OPC } & 5 & 5.11 & -21.78 & 3.50 \\ \text { 100nm WPC } & 5 & 5.07 & -23.44 & 0.98 \\ \text { 100nm WPC no salt } & 7 & 4.50 & -41.19 & 8.08 \\ \text { 100nm WPC pH=2 } & 2 & 2.07 & -14.58 & 1.33 \\ \text { 100nm WPC MV soak } & 2 & 2.16 & -17.83 & 1.64 \\ \text { 100nm WPC MV soak } & 5 & 5.12 & -27.76 & 1.08 \\ \text { 10nm OPC } & 5 & 5.15 & -17.38 & 0.55 \\ \text { 10nm OPC (no pvp) } & 5 & 5.16 & -28.92 & 3.38 \\ \text { 15nm Belgian } & 5 & 5.15 & -38.85 & 4.51 \\ \text { 15nm WPC } & 5 & 5.13 & -22.43 & 0.46 \\ \text { Anodisk } & 5 & 5.17 & 17.30 & 0.31 \\ \text { SLIP on 10nm OPC } & 5 & 5.17 & -32.21 & 0.27\end{array}$

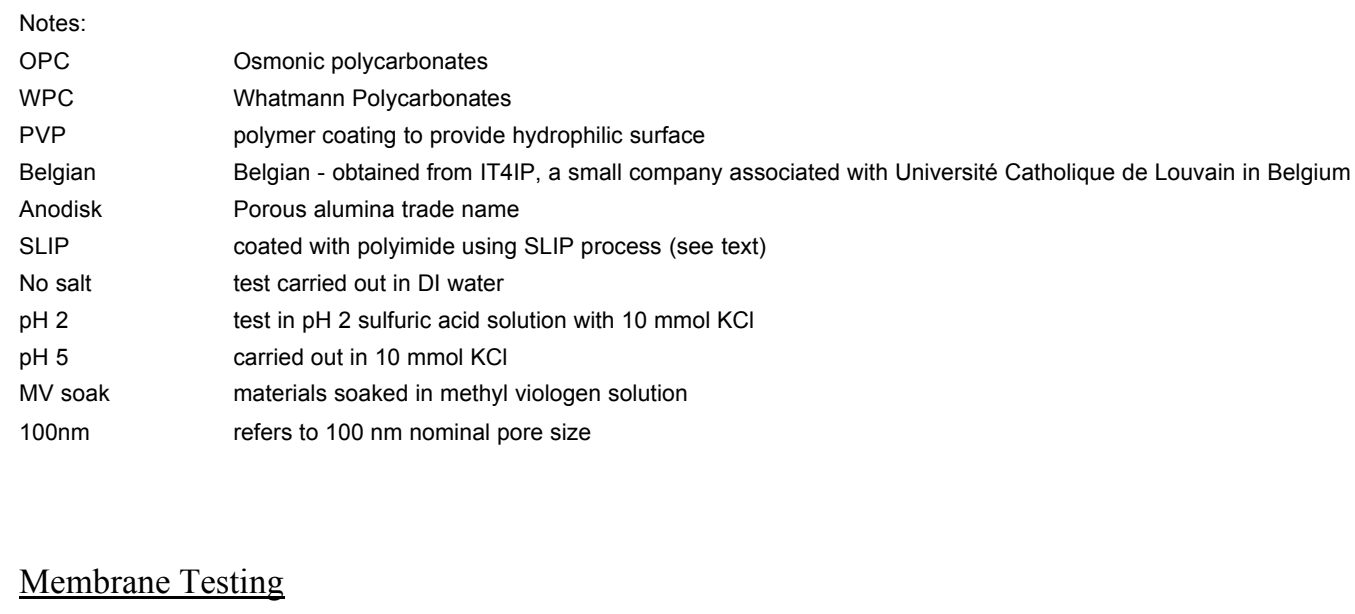

$\underline{\text { Membrane Testing }}$

The membranes were tested to determine their ion transport and permselectivity properties. In general, the tests of our fabricated membranes were run side by side with commercial electrodialysis membranes. Membranes obtained from GE-Ionics and Asahi were used for these | comparison tests. 
A membrane testing apparatus was constructed that connects two chambers separated by a membrane or pair of membranes. The chambers were filled with the desired salt solution, generally a dilute sodium nitrate solution. A fixed DC voltage or current was applied to the two plates, and the reciprocal (current or voltage) measured. Voltages between 2 and 10 volts were used in the tests. The voltage had to be high enough to sustain an electrode reaction (water electrolysis), but was preferred to be low to minimize energy loss associated with the electrode reactions and gas generation (hydrogen and oxygen). If there were no electrode reaction, ions would be shielded from the electrostatic field through double layer formation, and no ion transport would take place. The ion transport flux was determined by measuring solution conductivity over time. Comparison of measured current (from a meter) and ion current (from

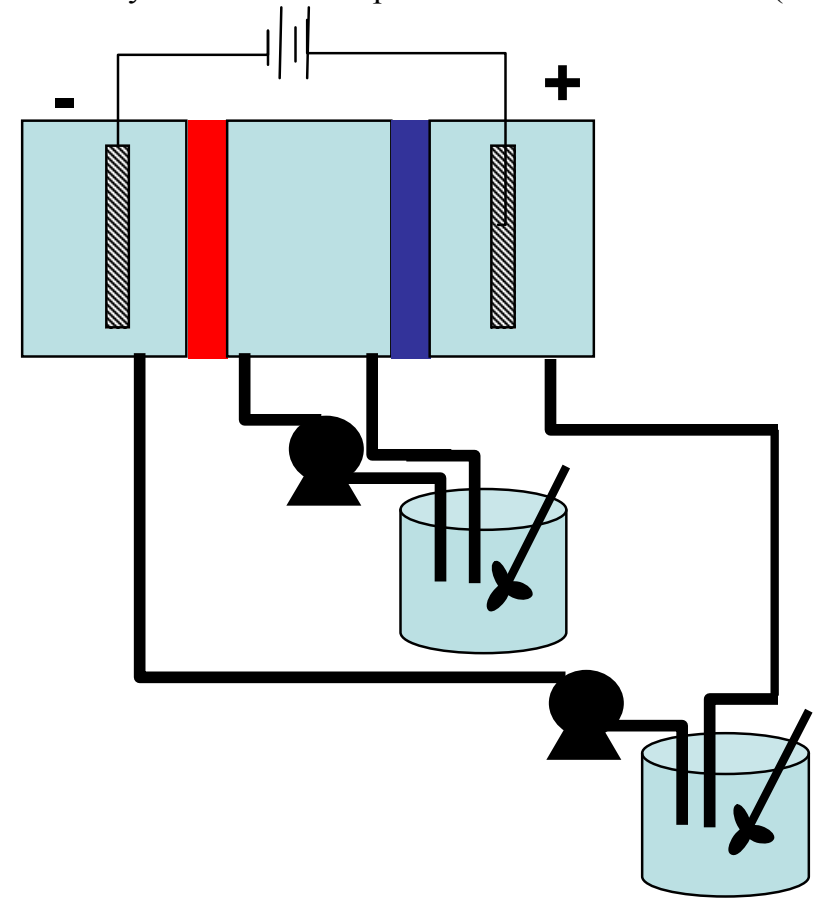

Figure 8. Schematic of electrodialysis test cell. The cation (red) and anion (blue) membranes lie between the anode and cathode used to supply a DC field to drive ion transport through the membranes. Fluids in all compartments were continually circulated. the measured change in solution conductivity) provided a measure of current efficiency. In addition, because of electrolysis reactions at the electrodes, $\mathrm{pH}$ was monitored in all compartments. Fluids from the cathode and anode were mixed to neutralize the acid generated at the anode and the base generated at the cathode.

The tests were carried out using either a commercial membrane paired with a nanoporous membrane, two commercial membranes, or two nanoporous membranes. Over 70 different tests were performed to examine the performance of a variety of different membrane configurations. Typical test results are shown in Figure 9. The conductivity and the nitrate level were monitored as a function of time. The slope of this first graph yielded the change in nitrate level as a function of time (ppm Nitrate/hr). If this same test was

done under different voltages, the rate of change of the nitrate as a function of time could then be plotted as a function of power $(\mathrm{mW})$. The slope of this second curve enables the determination of the ppm Nitrate separated per $\mathrm{mWhr}$.

The test results are interpreted and discussed in more detail in Bourcier et al. (2006). Our most significant conclusion is that nanoporous membranes do function as cation-anion permselective $\underline{\text { membranes with higher energy efficiency than commercial membranes. The test results shown in }}$ 


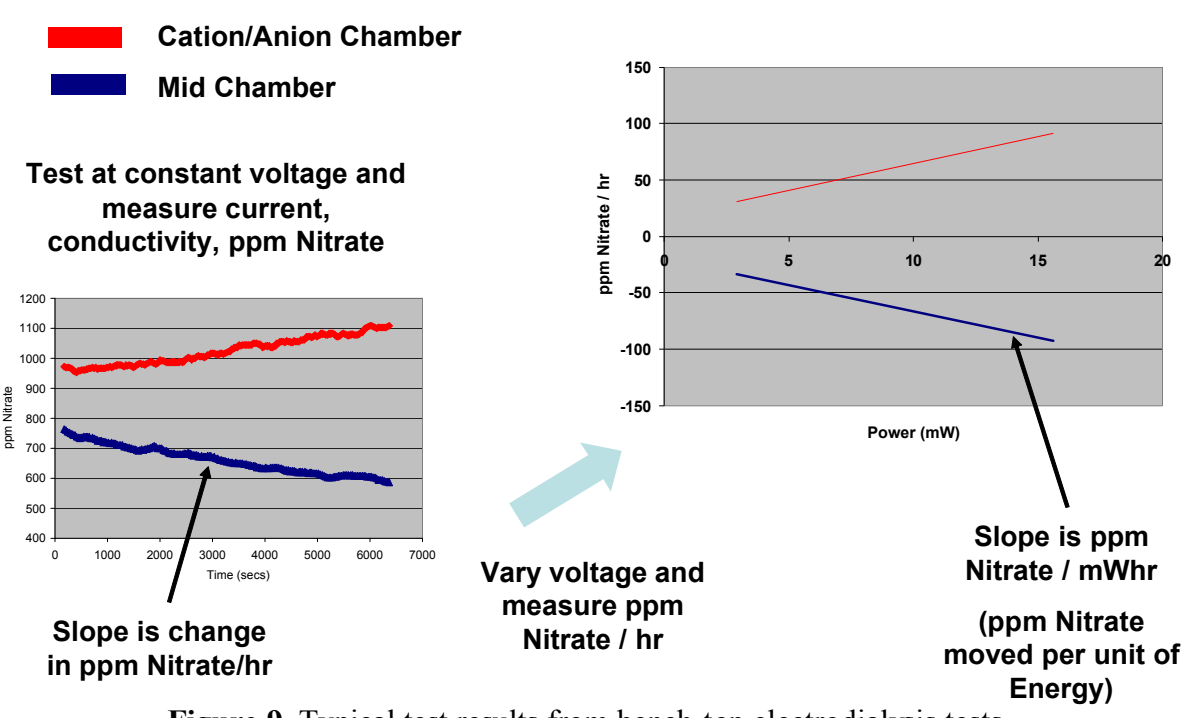

Figure 9. Typical test results from bench-top electrodialysis tests.

Test results generated data for energy use for nitrate removal.

Figure 9 shows that the membranes are slightly more energy efficient than commercial electrodialysis membranes. Table 1 shows a number of other advantages that the nanoporous membranes have over existing commercial membranes.

There is significant room for improved energy efficiency in nanoporous membranes. This is because the commercial nanoporous membranes that were tested had a pore density of $10^{9}$ pores $/ \mathrm{cm}^{2}$ or less. Maximum pore densities that could be fabricated for $10 \mathrm{~nm}$ pores are about 100 times higher. Energy efficiency is likely to be linearly proportional to pore density, up to the point where ion transport is limited by concentration polarization. Concentration polarization refers to the situation where the concentration of ions near the membrane surface is depleted due to ion transport though the membranes, and the ion flux therefore decreases. Work to determine the relative energy efficiency of higher pore density membranes was not carried out in this LDRD project and will be pursued with proposals for outside funding

Table 2. Comparison of nanoporous electrodialysis membranes to commercial electrodialysis membranes

\begin{tabular}{|c|c|c|}
\hline & $\begin{array}{c}\text { Commercial ED } \\
\text { Membrane }\end{array}$ & $\begin{array}{c}\text { Nanoporous } \\
\text { Membranes }\end{array}$ \\
\hline $\begin{array}{c}\text { Energy Efficiency: } \\
\text { ppm Nitrate / mWhr }\end{array}$ & 4.7 & $5.2^{*}$ \\
\hline Permselectivity (Diode Effect) & Excellent & Excellent \\
\hline Thickness (microns) & 1,000 & 6 \\
\hline Must be stored wet & Yes & No \\
\hline $\begin{array}{c}\text { Maximum Temperature of } \\
\text { Operation }\end{array}$ & $\mathbf{5 0}^{\circ} \mathrm{C}$ & $\mathbf{1 4 0}^{\circ} \mathrm{C}$ \\
\hline
\end{tabular}

* For $10^{9}$ pores $/ \mathrm{cm}^{2}$. Membranes with $10 \mathrm{~nm}$ pores can be fabricated with up to $10^{11}$ pores $/ \mathrm{cm}^{2}$, further increasing energy efficiency.
Deleted: The test results are interpreted and discussed in more detail in Bourcier et al. (2006). Our most significant conclusion is that nanoporous membranes do function as cation-anion permselective membranes with higher energy efficiency than commercial membranes. The test results shown in Table 1 demonstrate that the membranes are slightly better in energy efficiency when compared to commercial electrodialysis membranes. Table 1 shows a number of other advantages that the FIC membranes have over existing commercial membranes. 
Some tests were also performed using a single membrane, instead of a membrane pair. In this geometry, because only cations or anions pass through the membrane, a charge imbalance develops. This effect will act to slow further ion transport through the membrane. These tests therefore are most useful to compare membranes in short duration tests, and to measure membrane anion versus cation permselectivity. They are less useful for determining membrane performance data, such as energy efficiency. Results of single membrane tests that compare commercial membranes with nanoporous membranes are shown in Figure 10.
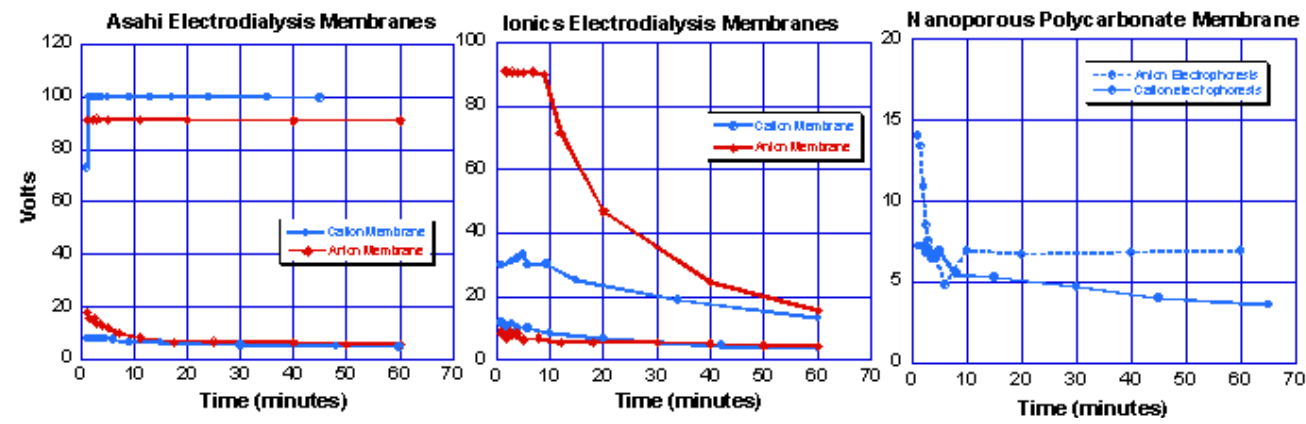

Figure 10. Single membrane test results that compare voltages needed to drive one milliamp of current through commercial membranes (Asahi and Ionics) and a nanoporous polycarbonate (not functionalized) membrane. A smaller voltage is needed to drive the same ion flux through the nanoporous membranes indicates a higher energy efficiency, although the permselectivity is not as great as with the commercial membranes.

\section{$\underline{\text { Investigations of pore gating mechanisms }}$}

As part of this project, we investigated the nature of gating of ion transport through pores. The question is how to best engineer the electrostatic field around pore entrances to: (1) allow ion transport and restrict water transport; (2) to selectively allow either cation or anion transport; and (3) to selectively allow certain species to pass through the pore while restricting the passage of others. In the later mode the pore functions similarly to ion channels in cell walls that regulate the ion contents of cellular fluids. Optimization of selective ion transport through pores is a key need for using nanoporous membranes for electrodialysis treatment of impaired waters.

In order to investigate gating, we carried out a matrix of measurements of transport rates of charged species through pores having varying sizes and surface charges. In order to use commercially available nanoporous membranes (PCTE) that have pores sizes too large to sterically or electrostatically restrict small inorganic ion (e.g. $\left.\mathrm{Na}^{+}, \mathrm{Cl}^{-}, \mathrm{NO}_{3}{ }^{-}\right)$transport, we used sodium and chloride salts of relatively large charged organic species as electrolytes in the tests (Fig. 8); methyl viologen chloride as the salt having a large cation $\left(\mathrm{MV}^{2+}\right)$, and sodium naphthalene disulfonate as the salt of a large anion $\left(\mathrm{NDS}^{2-}\right)$. Membranes with nominal pore sizes of $15,100,400$, and $800 \mathrm{~nm}$ were used in the tests. Our idea was that by using a larger ion, we should be able to investigate gating phenomena with commercially available membrane pore sizes. Tests were conducted at $\mathrm{pH} 2$, where the membranes had a positive surface charge, and $\mathrm{pH}$ 
5, where the membranes had a negative surface charge, as determined from streaming potential measurements described in the literature.

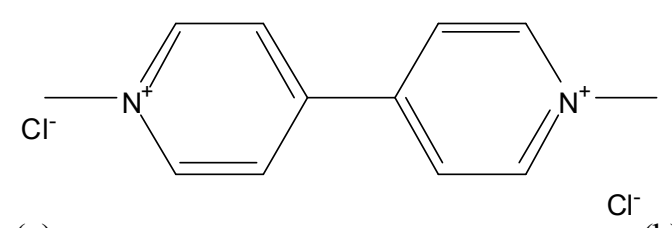

(a)

(b)

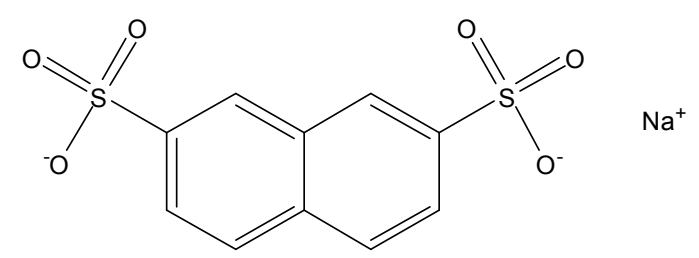

Figure 8. Structures of organic ions used in pore gating experiments; (a) methyl viologen and (b) naphthalene disulfonate.

We anticipated that for the salinities and $\mathrm{pH}$ conditions of the tests, gating effects would only be observed for the smallest pore sized membranes, because there would be no size exclusion at the larger pore diameters, and any electrostatic effects should be localized to within $10-20 \mathrm{~nm}$ of the

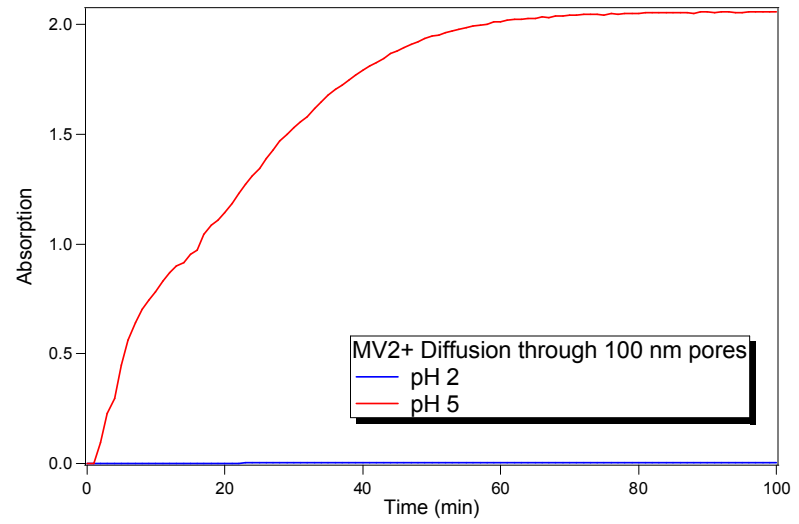

Figure 9. Diffusive transport of methyl viologen (10 mmolar solution as chloride salt) through $100 \mathrm{~nm}$ pores in polycarbonate for positive $(\mathrm{pH} 2)$ and negative $(\mathrm{pH} 5)$ surface. Diffusion is rapid when polycarbonate has negative surface charge but is essentially zero for positive surface charge. pore walls based on the double layer thickness for the ionic strengths of our test solutions. Free passage of the salts should occur for $100 \mathrm{~nm}$ and greater pore sizes. However, our experiments showed clear gating related to surface charge of the membrane at $100 \mathrm{~nm}$ pore sizes. Gating was measurable even for pore sizes as large as $800 \mathrm{~nm}$. This unexpected result is described in (Letant et al., 2006) where modeling of ion transport through pores was carried out using the techniques described in next section. Modeling suggests that gating is due to electrostatic trapping of the more mobile ion (chloride in the case of methyl viologen salts) at the membrane surface, which

electrostatically blocks the diffusion of the $\mathrm{MV}^{2+}$ ions through the pores. The exclusion is only effective when the membrane surface charge is opposite of that of the more mobile ion. The gating disappears when the surface charge is negative. Electrostatically opposite results were found for the naphthalene disulfonate ion, which has a negative charge. Gating was found at $\mathrm{pH}$ 5 and free diffusion took place at $\mathrm{pH} 2$, opposite the result for $\mathrm{MV}^{2+}$.

This result was unexpected and may provide the basis for the development of new structures for selective gating of charged species using much larger pore diameters than previously believed 
possible. This would allow both greater throughput and the use of materials with pores sizes that are more easily fabricated than would be the case for smaller pore sizes with diameters comparable to double layer thicknesses.

\section{Ion pump concept}

An outgrowth of this project was a new idea for desalination using electrostatic sorption of ions onto charged plates. The idea arose during conversations with Dr. Edward Cussler (U.

Minnesota) during a discussion of capacitive deionization (CDI) while Dr. Cussler was visiting LLNL. CDI using carbon aerogel electrodes was developed at LLNL during the 1990s.

Ion pumping makes use of the immobilization of ions onto conductive charged surfaces when an electrical potential is applied. Figure 10 shows schematically how the process works.
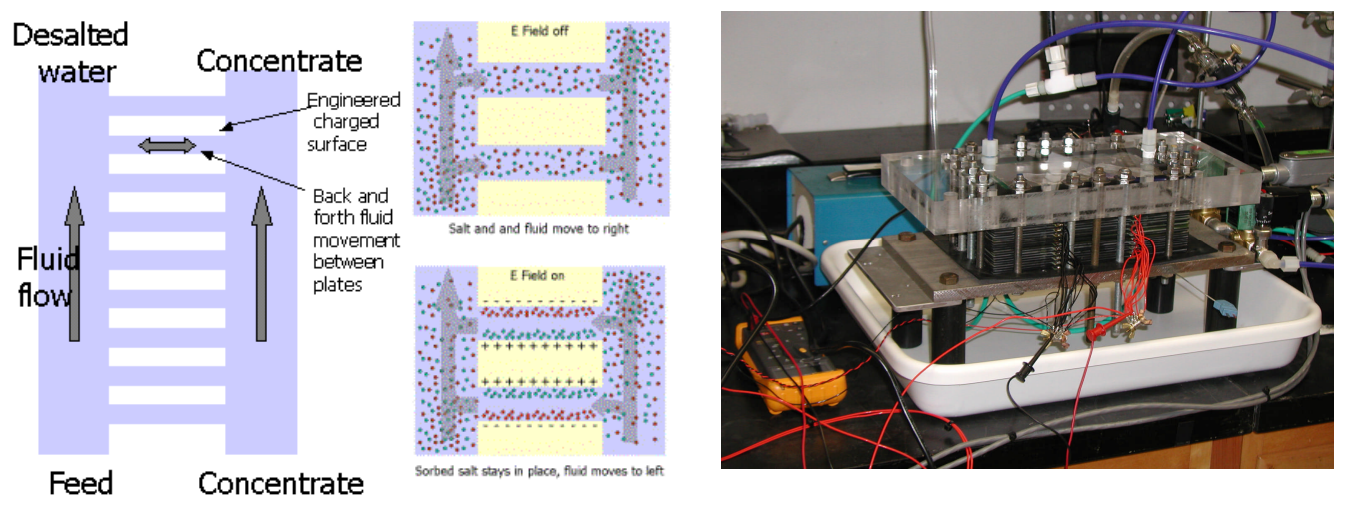

Figure 10. The ion pump concept (left) shows how salt can be removed from solution by using electrosorption in a novel back-and-forth flow geometry where flow and applied field are synchronized. Flow towards the right takes place with the field off, allowing ions to move with the fluids. Flow towards the left takes place with the field on, anchoring the ions in place on the charged surfaces. The net effect is to ratchet salt to the right through the horizontal flow channels into a concentrate stream that exists out the top right.

Desalinated fluid flows out the top left. Our first prototype ion pump is shown on the right.

A simple prototype ion pump was constructed and used to desalinate water. Tests showed proof of concept. The major issue at this time is maintaining uniform, back and forth fluid flow, and eliminating any entrained air bubbles.

This project has been funded externally by the California Department of Water Resources (Prop 50 grant, $\$ 997 \mathrm{~K}$ over 3 years). Work will begin after a contract has been approved. A record of invention (ROI-11609) was filed and a patent application is in progress (DOE Case No. S-

108,256). 


\section{Modeling}

In reviewing the literature describing existing membrane-based water treatment technologies, including both selective extractions and desalination, it became clear that the development of new technologies and the optimization of existing technologies could benefit greatly from an improved understanding of ion transport through membranes. In order to simulate transport through nanoporous membranes, a critical need existed for a numerical method to solve the Poisson-Boltzmann equation in the vicinity of charged nanostructured materials. This would allow us to correctly calculate the distribution of ions in the near-surface region of a membrane where the electrical double layer exists, and outwards to bulk solution. When coupled to numerical solutions of the Navier-Stokes equation for fluid flow, and a Langevin methodology for diffusion, we would have a powerful tool for investigating new approaches to desalination and selective extractions for water treatment. Through a close coupling of computations to experiments, these simulations could provide the science-driver for investigations of a variety of proposed separations technologies.

\section{Calculation of electrostatic potentials in the vicinity of nanostructures}

We developed a method for calculating the electrostatic potentials and fields in the vicinity of geometrically complex engineered nanostructures in electrolytes of arbitrary $\mathrm{pH}$ and ionic strength (Schaldach et al., 2004). The method involves direct summation of charged DebyeHückel spheres comprising the nanostructured surfaces and, by including charge redistribution on the surface of conducting materials held at constant potential, is applicable to mixed boundary conditions. The method was validated by comparison to analytical solutions for an infinite plane (Gouy-Chapman), an infinite cylinder (Bessel functions), and an infinite plane which contains a hole and which is held at constant potential. Excellent agreement between the potentials obtained by our numerical method and the closed form solutions was found for these conditions.

We applied our method to the calculation of the electric field enhancement in the vicinity of a nanoporous membrane in the case (1) where pore wall is held at constant charge and case (2) where the pore wall is held at constant potential. In case (2) the electric field was found to be enhanced by the charge buildup in the rim of the pore, which redistribution results from the potential being held constant in the conducting region. Ion concentrations are also calculated; positive ion rejection is found to be enhanced by this charge buildup in the region of the rim when a constant positive potential is applied. This work is described in detail in Schaldach (2004). This work provides the basis for designing nanoporous membranes that are permselective to ion transport and species selective when functionalized.

\section{Electrostatic properties of ions in solution}

Molecular ions in solution are known to induce surface polarization charge at the moleculesolvent interface, the solvent accessible surface, of the ion. These charges are due to the discontinuity in dielectric constant at the interface and are a function of the ionic strength of the medium in which they reside. Our method incorporates these solvent effects, including ionic 
strength, into $\underline{a b} \underline{i n i t i o}$ electronic structure determinations (Wilson et al., 1997) ${ }^{2}$. This Green's function-based method couples the Schrödinger and linearized Poisson-Boltzmann equations through both the single and double layer surface charge distributions induced at a molecularsolvent interface. Single and double layers of charge are obtained from solution of the PoissonBoltzmann equation (and added iteratively to the Hamiltonian) using electronic structure results (MP2/6-31G**) directly, eliminating the uncertainty in employing fractional charges. We find the iterative method to converge in only a few $(\sim 2-4)$ "complete" iterations; the determination of self-consistent-field (SCF) wave functions for a given set of background charges involves, perhaps, tens of "micro" HF iterations. These HF wave functions are then employed to obtain an MP2 energy and also as the source charge distribution for the next Poisson-Boltzmann solution. In this way, the electrochemical double layer of the ions to be separated is well-described. This methodology developed previously by W. Wilson was available at the start of this project and was incorporated into our modeling approach. It was used to populate the dielectric continuum with discrete ions having solvent accessible surfaces that correctly account for the properties of the media in which they are embedded.

\section{Langevin Dynamics}

Athough modeling of membrane transport processes could be carried out using rigorous ab initio molecular dynamics methods, we used a statistically-based Langevin method to compute molecular transport. Unlike traditional molecular dynamics approaches which must be integrated on a time scale of order $10^{-15}$ seconds, solutions to Langevin dynamical equations of motion are integrated on a $10^{-4}$ second time scale. The information lost as a result includes the vibrational and stretching modes of the molecule and the details of the collisions with water molecules, treated in the Langevin method by statistical mechanics (Brownian motion). This information is not critical for our present purpose. These stochastic differential equations include deterministic forces due the motion of the charged ion in solution in the presence of an electric field (electrophoresis), electroosmotic and pressure-driven fluid flow, viscous forces which depend on the viscosity at the boundary layer of the solution and ion-electrostatic steering effects due to charges and potentials on the membrane. The equations also take account of stochastic effects such as translational and rotational diffusion.

A modeling code that embodies the capabilities described above was developed as part of this project in order to investigate the motion of solute particles (ions) embedded in a continuous dielectric medium in the vicinity of or traveling through a nanopore. We used the code to carry out computations of the permselectivity of nanopores due to double layer effects, ion mobilities related to movements of ions in the ion pump, and electrostatic gating of pores.

In addition, the code was used to calculate the dielectrophoretic forces on nanoparticles in fluid environments where variations in the electric field and electric field gradients are on the same size scale as the particle. This phenomena could potentially underlie an important new separations method for molecular and ionic species analogous to dielectrophoretic separations technologies used to separate macromolecules.

\footnotetext{
${ }^{2}$ Wilson, W.L., C. M. Schaldach, and W. L. Bourcier (1997) Single and double layer coupling of Schrodinger and Poisson-Boltzmann equations. Chem. Phys. Letters 267:431-437.
} 
The Boundary Element Dielectrophetic Force (BEDF) method involves constructing a solventaccessible or molecular surface surrounding the particle, calculating the normal component of the electric field at the surface boundary elements and then solving a system of linear equations for the induced surface polarization charge on each element (Schaldach et al., 2004). Different surface elements of the molecule may experience quite different polarizing electric fields, unlike the situation in the point dipole approximation. A single $100 \AA$ radius ring test configuration was employed to facilitate comparison with the well-known point dipole approximation (PDA). We found remarkable agreement between the forces calculated by the BEDF and PDA methods for a $1 \AA$ A polarizable sphere. However, for larger particles, the differences between the methods become qualitative as well as quantitative; the character of the force changes from attractive at the origin of the ring for a $50 \AA$ sphere, to repulsive for a $75 \AA$ sphere. Equally dramatic differences are found in a more complex electrical environment involving two sets of 10 rings.

The results of these calculations provide design rules for developing nanostructures that could be used for molecular separations.

\section{Results/Technical Outcomes}

This project was targeted at providing a foundation for new more energy efficient and species selective water treatment technologies. Our goal was to develop a new type of membrane for use in electrodialysis for removal of nitrate and other contaminants from impaired water supplies. Our membrane development work was carried out in conjunction with work to develop and apply a numerical capability to model membrane transport. The modeling work provides a first test of potential membrane separation processes and allows a more efficient use of resources for fabrication and testing of new separations methods.

In this project, we demonstrated that nanoporous membranes can be used to desalinate water when employed in electrodialysis, confirming our hypothesis that double layer overlap should provide cation or anion permselectivity. As a result, a new generation of energy efficient and species selective electrodialysis technologies is now possible, that could potentially greatly lower the cost of treating 'impaired' waters that are common throughout the world.

In addition, we began work on an alternate desalination technology, the "ion pump", that also has the potential to significantly lower the energy consumption of desalination. The ion pump has an additional advantage in that it is very simple, uses commonly available materials, and for these reasons could have a major impact on fresh water availability in third world countries.

Finally, the computational methods developed as part of this project are now available for further developmental work on membrane separation processes of all kinds. The models were used in this project to provide the foundation for the development of new selective separations methods for electrodialysis, desalination through 'ion pumping', and molecular species separations based on dielectrophoretic forces. 


\section{Intellectual property (attached):}

IL-11609 "Desalination using electrostatic ion pumping"

Inventors: W. Bourcier, R. Aines, J. Haslam, C. Schaldach, and K. O’Brien, Patent Application, Date: $1 / 30 / 2006$.

IL-11301 "Computer designed nanoengineered materials for separation of dissolved species" Inventors: W. Wilson, C. Schaldach, W. Bourcier, and P. Paul

Date: 11/30/2003 (Patent Applied for)

IL-11462 "Hybrid approach for selective removal of contaminants"

Inventors: J. Haslam, W. Bourcier, and K. O’Brien

\section{Publications (attached):}

Letant, S. E, C. M. Schaldach, M. R. Johnson, A. Sawvel, W. L. Bourcier, and W. D. Wilson (2006) Evidence of gating in hundred nanometer diameter pores: An experimental and theoretical study. Sub. to Nanoletters (UCRL-JRNL-218429)

Schaldach, C. M., W. L. Bourcier, P. H. Paul, and W. D. Wilson (2004) Dielectrophoretic forces on the nanoscale. Langmuir 20:10744-10750. (UCRL-TR-203675)

Schaldach, C. M., W. L. Bourcier, P. H. Paul, and W. D. Wilson (2004) Electrostatic fields in the vicinity of engineered nanostructures. J. of Colloid and Interface Science 275:601-611 (UCRLTR-203010)

Bourcier, W. L., K. O’Brien, J. Haslam, and A. Sawvel (in prep) Electrodialysis using nanoporous membranes. For submission to J. Membrane Science.

\section{Exit Plan:}

Throughout the course of this project, we have made efforts to interact with all participants involved with water treatment, including local water utilities and water wholesalers, engineering companies, water technology OEMs, water associations, and governmental bodies such as the U.S. Bureau of Reclamation that are chartered to manage water resources and fund R\&D efforts for water treatment. We have partnered with several of these groups on proposals to various funding agencies such as the Water Reuse Foundation and American Water Works Association Research Foundation (AWWARF). For examples, partners on these proposals include: Zone 7 Water Agency, Dublin-San Ramon Services District, City of Modesto Public Works Department, City of Ripon, Boyle Engineering, Tetra Tech Corporation, and the U.S.B.R.

While this project was active, two new funding sources for subsequent R\&D work became available. The first is funded by the State of California through Proposition 50 passed in 2002. Prop 50 allocated over $\$ 600$ million dollars for water infrastructure development, including nearly $\$ 100$ million for technology development. We submitted several proposals to this call, two of which were funded, and one of which is still being considered. The proposals include: 
To Prop 50 RFP - Department of Health Services

Hybrid Electrodeionization for Selective Removal of Nitrate/Perchlorate Ions, J. Haslam P.I., \$1.8M (E\&E)

status: under review

A Pre-Concentrator for the Detection of Viruses in Water Supplies, C. Schaldach, P.I. (\$1.4M)

(E\&E)

status: not funded

Fast bio-organism detector for drinking water monitoring and alarms, S. Letant P.I., \$1.1M

(C\&MS)

status: not funded

To Prop 50 RFP - Department of Water Resources

Desalination Using Electrostatic Ion Pumping - Bourcier P.I., \$997K

Status: funded

Pilot / Demonstration Study of Field Induced Charged Membranes for Brackish Water

Desalination - O'Brien PI, \$1.8M

Status: not funded

Pajaro Watershed Groundwater Desalination Feasibility Study - partner with Santa Clara Valley Water District, O’Brien PI, \$100K

Status: funded

To ESTCP:

Field Induced Charged (FIC) Membranes for Selective Removal of Perchlorate Ions- partner with Edwards Airforce base - O'Brien PI, \$1.1M

Status: not funded

In addition, Senate Bill S. 1860 has authorized DOE to invest in research aimed at improving the energy efficiency of water treatment, and developing technologies to reclaim previously unusable water. These funds have not yet been authorized, but LLNL is now more strongly positioned, because of our LDRD investment, to compete for these funds should they become available. Similarly, H.R. 3182 in the House reauthorizes the Water Desalination Act of 1996 and increases its allocation to $\$ 75$ million (from $\$ 25$ million) with funds to be spent on the development of advanced water treatment technologies that lower the treatment costs for desalination and selective removal. The funds are to be administered by the Department of the Interior through the U.S.B.R. LLNL has established a strong working relationship with key 
U.S.B.R. staff and is well positioned to take advantage of this funding opportunity. These efforts are continuing.

\section{Attachments:}

\section{$\underline{\text { Records of Invention }}$}

(1) IL-11609 "Desalination using electrostatic ion pumping" Inventors: W. Bourcier, R. Aines, J. Haslam, C. Schaldach, and K. O’Brien, Patent Application, Date: $1 / 30 / 2006$.

(2) IL-11301 "Computer designed nanoengineered materials for separation of dissolved species" Inventors: W. Wilson, C. Schaldach, W. Bourcier, and P. Paul

Date: 11/30/2003 (Patent Applied for)

(3) IL-11462 "Hybrid approach for selective removal of contaminants"

Inventors: J. Haslam, W. Bourcier, and K. O'Brien

$\underline{\text { Journal Publications: }}$

(4) Letant, S. E, C. M. Schaldach, M. R. Johnson, A. Sawvel, W. L. Bourcier, and W. D. Wilson (2006) Evidence of gating in hundred nanometer diameter pores: An experimental and theoretical study. Sub to Nanoletters (UCRL-JRNL-218429-DRAFT)

(5) Schaldach, C. M., W. L. Bourcier, P. H. Paul, and W. D. Wilson (2004) Dielectrophoretic forces on the nanoscale. Langmuir 20:10744-10750. (UCRL-TR-203675)

(6) Schaldach, C. M., W. L. Bourcier, P. H. Paul, and W. D. Wilson (2004) Electrostatic fields in the vicinity of engineered nanostructures. J. of Colloid and Interface Science 275:601-611

(UCRL-TR-203010)

Funded Proposals:

(7) Desalination Using Electrostatic Ion Pumping - Bourcier P.I., \$997K, California Department of Water Resources. 